\title{
Intra-subject approach for gait-event prediction by neural network interpretation of EMG signals
}

\author{
Francesco Di Nardo ${ }^{1 *}$ (D), Christian Morbidoni ${ }^{1}$, Guido Mascia ${ }^{2}$, Federica Verdini ${ }^{1}$ and Sandro Fioretti ${ }^{1}$
}

\author{
*Correspondence: \\ f.dinardo@staff.univpm.it \\ ${ }^{1}$ Department of Information \\ Engineering, Università \\ Politecnica delle Marche, \\ via Brecce Bianche, \\ 60131 Ancona, Italy \\ Full list of author information \\ is available at the end of the \\ article
}

\begin{abstract}
Background: Machine learning models were satisfactorily implemented for estimating gait events from surface electromyographic (sEMG) signals during walking. Most of them are based on inter-subject approaches for data preparation. Aim of the study is to propose an intra-subject approach for binary classifying gait phases and predicting gait events based on neural network interpretation of sEMG signals and to test the hypothesis that the intra-subject approach is able to achieve better performances compared to an inter-subject one. To this aim, sEMG signals were acquired from 10 leg muscles in about 10.000 strides from 23 healthy adults, during ground walking, and a multi-layer perceptron (MLP) architecture was implemented.
\end{abstract}

Results: Classification/prediction accuracy was tested vs. the ground truth, represented by the foot-floor-contact signal provided by three foot-switches, through samples not used during training phase. Average classification accuracy of $96.1 \pm 1.9 \%$ and mean absolute value (MAE) of $14.4 \pm 4.7 \mathrm{~ms}$ and $23.7 \pm 11.3 \mathrm{~ms}$ in predicting heel-strike (HS) and toe-off (TO) timing were provided. Performances of the proposed approach were tested by a direct comparison with performances provided by the inter-subject approach in the same population. Comparison results showed $1.4 \%$ improvement of mean classification accuracy and a significant $(p<0.05)$ decrease of MAE in predicting HS and TO timing (23\% and 33\% reduction, respectively).

Conclusions: The study developed an accurate methodology for classification and prediction of gait events, based on neural network interpretation of intra-subject sEMG data, able to outperform more typical inter-subject approaches. The clinically useful contribution consists in predicting gait events from only EMG signals from a single subject, contributing to remove the need of further sensors for the direct measurement of temporal data.

Keywords: Surface EMG, Machine learning, Neural networks, Gait-phase classification, Ground walking, Intra-subject data

\section{Background}

Gait analysis is acknowledged as the main approach for quantitatively assessing the alteration of motor function in different contexts, such as in basic research and clinics. Technological development is making available smart and wearable sensors (inertial 
measurement units, IMUs) and robust artificial intelligence methods for handling large amount of data and signals (machine and deep learning). This kind of innovation is starting to allow a reduction of the complexity of experimental protocols for gait analysis and a cheaper, less-invasive, and more comfortable assessment of gait data. This is particularly true for the problem of assessing gait temporal parameters and events, such as stride length and the timing of heel-strike (i.e., the instant when the foot touches the ground) and toe-off (i.e., the instant when the foot-toes clear the ground). In many instances, IMUs showed to be convenient and reliable for an ecological (out of the laboratory) assessment of walking parameters [1]. However, it has been reported that EMGbased approach seems to be preferable over different approaches (including IMU), for specific environments such as control of exoskeleton devices [2], where EMG signals could be used to identify segment motion in advance, thus limiting delays in control action. An example of this is reported by Wentink et al. [3] who showed that the analysis of EMG signals allows assessing gait initiation earlier $(63-138 \mathrm{~ms})$ than inertial sensors, in a population of transfemoral amputees. In this and other cases, EMG-based approach seems to be really valuable [4-6]. Moreover, in the analysis of the majority of neuromuscular diseases, such as for example spastic cerebral palsy, the acquisition of sEMG signals is essential. Thus, the possibility of assessing HS and TO timing directly from sEMG signal, without using additional sensors such as foot-switch and IMUs, seems to be very useful in those cases.

Machine learning approaches were also satisfactorily implemented for the estimation of gait events from both kinematic data [2, 7-9] and electromyographic (EMG) signals [9-12] during walking. The success of machine learning approaches has opened a novel perspective for reducing the complexity of experimental set-up. Predicting gait events from only EMG signals could remove the need of further sensors or systems (foot-switch sensors, pressure mats, IMUs, stereo-photogrammetry [1, 13-15]) for the direct measurement of temporal data. This would be particular suitable for specific fields where measuring myoelectric signals is strongly recommended, such as the analysis of neuromuscular diseases or for walking-aid devices $[16,17]$.

Only few efforts in this direction $[11,12]$ proved to be able in providing a reliable classification of swing and stance phases and an accurate prediction of heel-strike (HS) and toe-off (TO), with mean errors comparable to those reported for IMU-based studies [1]. Nazmi et al. [11] fed EMG-based features to a single hidden layer neural network, reporting for unseen subjects a mean classification accuracy of $77 \%$ and mean absolute error of 35 and $49 \mathrm{~ms}$ in assessing HS and TO, respectively. The present group of researchers interpreted EMG signals by means of a multi-layer perceptron classifier with the aim of predicting HS and TO [12]. Outcomes on unseen subjects were promising, showing a mean classification accuracy of $93.4 \%$ and a mean accuracy error of $21 \mathrm{~ms}$ for HS and $38 \mathrm{~ms}$ for TO. These studies are based on the so-called inter-subject approach for data preparation, which consists in training the neural network with EMG data acquired during different strides of a population of homogeneous subjects and then testing the network on a population of brand new subjects $[11,12]$. Moreover, to measure classification performances also for learned subjects (i.e., subject included only in training phase), the training phase was further split into two subsets: training set containing the first part of each subject signal and the test set including brand new strides (not used for training) 
taken from the subjects involved in training. Both studies indicated that classification performances were better for learned subjects than for unseen ones, in terms of accuracy $(+10 \%$ in [11] and $+1.5 \%$ in [12]) and standard deviations (SD). Larger variability reported for unseen subjects seems to indicate that walking patterns could be very different from subject to subject, making the classification harder in subjects never seen before.

All these results and considerations raise the issue if a totally intra-subject approach could drive to a better performance in gait-phase classification. With intra-subject approach, we mean training the neural network with EMG data acquired during different strides of a single subject and then testing the network on brand new strides of the same subject. The few and preliminary studies reporting classification results based on the intra-subject approach, including our own, seem to support this hypothesis [10, 18-22]. Meng et al. [10] applied hidden Markov models to EMG signal to identify stance and swing phases, reaching a classification accuracy of 91\%. Ziegier et al. [19] reported a best-case accuracy of around $96 \%$ in the classification of gait phases by training a support vector classifier with EMG signals. To the same purpose, Joshi et al. [20] used Bayesian Information Criteria along with some standard feature extraction methods and Linear Discriminant Analysis classification algorithm. Best individual accuracies were around 94\%. Population involved in these three preliminary studies included two subjects at most. Also our preliminary reports showed encouraging results, characterized by mean intra-subject classification accuracy of $95.2 \pm 1.6 \%$ [18]. None of the above-mentioned studies $[10,18-20]$ attempted to assess gait events.

Thus, the purpose of the present study is (1) to propose an intra-subject approach for binary classification and gait-event prediction; (2) to test the hypothesis that an intrasubject approach is able to achieve better performances in gait phase classification compared to an inter-subject one and to extend this hypothesis to gait-event prediction. To this aim, an intra-subject approach was implemented for neural network interpretation of 10 surface electromyographic (sEMG) signals collected in 23 subjects. To test our hypothesis, a direct comparison was performed with classification and prediction performances provided by the inter-subject approach in the same population.

\section{Results}

The inter-subject approach was considered as the benchmarking approach, since it was validated in [12]. In the present study, it was applied to 10 sEMG signals (five per leg) acquired from 23 adults. The inter-subject approach implemented here in unseen subjects improves the performances reported in [12] both in terms of classification accuracy, $94.7 \pm 2.3 \%$ vs. $93.4 \pm 2.3 \%$, and prediction error, $H S-M A E$ : $18.7 \pm 7.8 \mathrm{~ms}$ vs. $21.6 \pm 7.0 \mathrm{~ms}$; TO-MAE: $35.1 \pm 20.0 \mathrm{~ms}$ vs. $38.1 \pm 15.2 \mathrm{~ms}$. Moreover, accuracy in each single subject does not fall below 90\%, except for a single subject (subject 4, 88.9\%). Best-case accuracy is $98.0 \%$ (subjects 18 and 23). Mean inter and intra-subject accuracies of gait-phase classification were compared in every subject in Table 1. Mean values over population (23 subjects) are shown in the last row of the table. The direct comparison between mean values showed an improvement of 1.4 points of the classification accuracy provided by the intra-subject approach (96.1 $\pm 1.9 \%)$, compared with the inter-subject approach $(94.7 \pm 2.3 \%)$. Besides gait-phase classification, the present study 
Table 1 Stance vs. swing classification accuracy provided by inter and intra-subject approach

\begin{tabular}{|c|c|c|c|c|}
\hline \multirow[t]{3}{*}{ Subject } & \multicolumn{4}{|c|}{ Classification accuracy (\%) } \\
\hline & \multicolumn{2}{|c|}{ Inter-subject } & \multicolumn{2}{|c|}{ Intra-subject } \\
\hline & Train & Test & Train & Test \\
\hline 1 & 96.9 & 97.7 & 97.5 & 97.5 \\
\hline 2 & 96.0 & 94.4 & 97.9 & 96.8 \\
\hline 3 & 95.6 & 94.8 & 96.5 & 95.7 \\
\hline 4 & 96.9 & 88.9 & 95.1 & 94.3 \\
\hline 5 & 97.2 & 93.9 & 97.8 & 97.8 \\
\hline 6 & 96.8 & 94.9 & 97.4 & 97.0 \\
\hline 7 & 96.8 & 95.5 & 98.1 & 97.6 \\
\hline 8 & 97.0 & 94.7 & 97.9 & 97.3 \\
\hline 9 & 96.8 & 94.0 & 97.1 & 95.9 \\
\hline 10 & 96.1 & 92.7 & 94.8 & 97.0 \\
\hline 11 & 96.8 & 92.2 & 93.0 & 93.1 \\
\hline 12 & 97.3 & 92.6 & 97.5 & 96.4 \\
\hline 13 & 97.1 & 94.3 & 96.2 & 96.9 \\
\hline 14 & 96.8 & 94.7 & 96.5 & 96.6 \\
\hline 15 & 96.7 & 94.9 & 97.2 & 97.0 \\
\hline 16 & 96.9 & 96.5 & 97.9 & 97.5 \\
\hline 17 & 97.2 & 93.8 & 98.2 & 98.1 \\
\hline 18 & 96.7 & 98.0 & 97.5 & 97.5 \\
\hline 19 & 96.7 & 97.8 & 97.9 & 96.8 \\
\hline 20 & 96.7 & 97.8 & 96.5 & 95.7 \\
\hline 21 & 97.4 & 91.3 & 97.1 & 90.3 \\
\hline 22 & 97.9 & 94.3 & 97.3 & 91.9 \\
\hline 23 & 97.1 & 98.0 & 90.8 & 95.2 \\
\hline Mean \pm SD & $96.8 \pm 0.5$ & $94.7 \pm 2.3$ & $96.7 \pm 1.8$ & $96.1 \pm 1.9$ \\
\hline
\end{tabular}

also provides a prediction of foot-floor-contact signal and a consequent assessment of HS and TO events. An example of predictions of foot-floor-contact signal provided by both inter and intra-subject approaches is reported in Fig. 1. Detailed comparison of the performance achieved with inter and intra-subject approaches is reported in Tables 2 and 3 for HS and TO prediction, respectively. A significant mean reduction of mean absolute error $(M A E)$ was detected in the prediction of HS provided by the intra-subject approach, compared with the inter-subject approach $(18.7 \pm 7.8 \mathrm{~ms}$ vs. $14.4 \pm 4.7 \mathrm{~ms}$; $23 \%$ reduction, $p<0.05)$. In the same way, a significant mean reduction of $M A E$ was detected in the prediction of TO provided by the intra-subject approach, compared with the inter-subject approach ( $35.1 \pm 20.9 \mathrm{~ms}$ vs. $23.7 \pm 11.3 \mathrm{~ms}$; $33 \%$ reduction, $p<0.05)$. No further significant differences were detected between groups.

\section{Discussion}

The present study was designed to test the hypothesis that an intra-subject approach is able to achieve better performances in terms of stance vs. swing classification and HS and TO timing assessment, compared to an inter-subject one. A multi-layer perceptron (MLP) architecture with three hidden layers composed of 512, 256 and 128 neurons, 

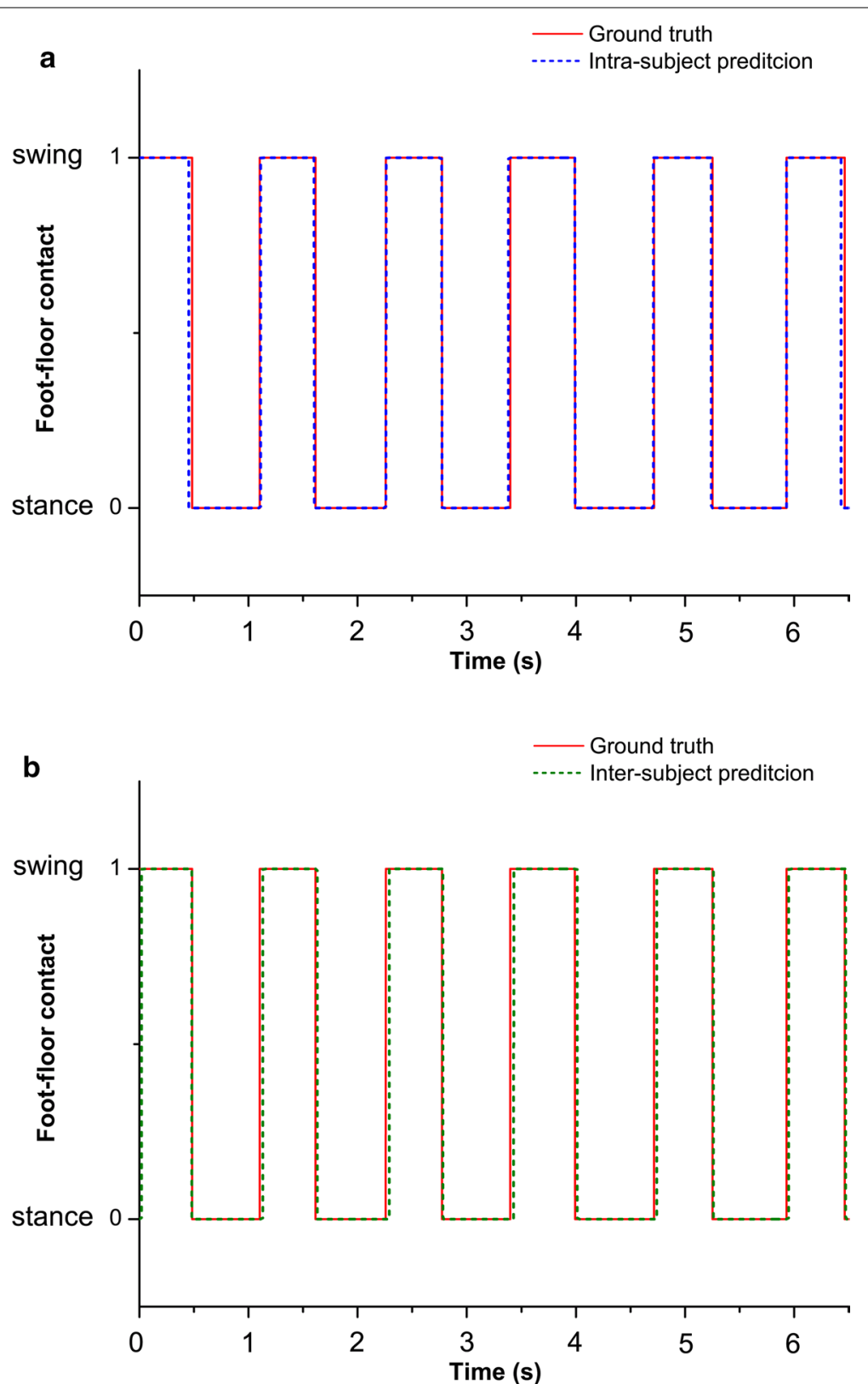

Fig. 1 Example of predictions of foot-floor-contact signal in the same six strides of a representative subject (subject 8), provided by intra (a) and inter (b) subject approaches

respectively, and a one-dimensional output was implemented to this aim. The intrasubject approach is articulated as follows: training the neural network with sEMG data measured during around 450 strides of one single subject and then testing the network on around 50 brand new strides of the same subject. The procedure was performed ten times, each time using a different slot as test set (tenfold cross-validation). Results were reported as average value over the tenfold. The present approach was able to provide a very precise classification of gait phases (Table 1, column "intra"), represented by mean $( \pm \mathrm{SD})$ accuracy over 23 subjects of $96.1 \pm 1.9 \%$ and supported by the fact that accuracy in each single subject does not fall below $90.3 \%$ (subject 21 ). Best-case accuracy is $98.1 \%$ 
Table 2 MAE (mean absolute error), precision, recall, and F1-score provided by inter and intra-subject approach for Heel-Strike (HS) prediction

\begin{tabular}{|c|c|c|c|c|c|c|c|c|}
\hline \multirow{2}{*}{$\begin{array}{l}\text { HS } \\
\text { Subject }\end{array}$} & \multicolumn{2}{|c|}{ MAE (ms) } & \multicolumn{2}{|c|}{ Precision } & \multicolumn{2}{|l|}{ Recall } & \multicolumn{2}{|c|}{ F1-score } \\
\hline & Inter & Intra & Inter & Intra & Inter & Intra & Inter & Intra \\
\hline 1 & 14.7 & 11.5 & 1.00 & 1.00 & 1.00 & 1.00 & 1.00 & 1.00 \\
\hline 2 & 29.7 & 17.3 & 0.99 & 1.00 & 0.99 & 0.99 & 0.99 & 1.00 \\
\hline 3 & 19.6 & 17.4 & 0.97 & 1.00 & 0.99 & 1.00 & 0.98 & 1.00 \\
\hline 4 & 12.8 & 10.3 & 1.00 & 1.00 & 1.00 & 0.99 & 1.00 & 1.00 \\
\hline 5 & 26.4 & 8.5 & 1.00 & 1.00 & 1.00 & 0.99 & 1.00 & 0.99 \\
\hline 6 & 11.5 & 7.4 & 1.00 & 1.00 & 1.00 & 0.99 & 1.00 & 0.99 \\
\hline 7 & 19.0 & 16.6 & 1.00 & 1.00 & 1.00 & 0.99 & 1.00 & 1.00 \\
\hline 8 & 25.6 & 16.2 & 0.99 & 1.00 & 1.00 & 0.99 & 1.00 & 1.00 \\
\hline 9 & 19.9 & 15.5 & 1.00 & 1.00 & 1.00 & 1.00 & 1.00 & 1.00 \\
\hline 10 & 27.5 & 16.1 & 0.99 & 1.00 & 0.99 & 0.99 & 0.99 & 0.99 \\
\hline 11 & 9.3 & 9.2 & 1.00 & 1.00 & 0.98 & 0.98 & 0.99 & 0.99 \\
\hline 12 & 8.0 & 6.8 & 1.00 & 1.00 & 1.00 & 0.99 & 1.00 & 0.99 \\
\hline 13 & 22.0 & 16.3 & 1.00 & 1.00 & 0.98 & 0.99 & 0.99 & 0.99 \\
\hline 14 & 20.3 & 15.0 & 1.00 & 1.00 & 1.00 & 1.00 & 1.00 & 1.00 \\
\hline 15 & 19.3 & 13.1 & 1.00 & 1.00 & 1.00 & 1.00 & 1.00 & 1.00 \\
\hline 16 & 15.3 & 10.6 & 1.00 & 1.00 & 1.00 & 1.00 & 1.00 & 1.00 \\
\hline 17 & 36.5 & 9.2 & 1.00 & 1.00 & 1.00 & 1.00 & 1.00 & 1.00 \\
\hline 18 & 10.8 & 11.5 & 1.00 & 1.00 & 1.00 & 1.00 & 1.00 & 1.00 \\
\hline 19 & 10.8 & 17.3 & 1.00 & 1.00 & 1.00 & 0.99 & 1.00 & 1.00 \\
\hline 20 & 11.7 & 17.4 & 1.00 & 1.00 & 1.00 & 1.00 & 1.00 & 1.00 \\
\hline 21 & 21.3 & 23.1 & 0.98 & 0.95 & 0.97 & 0.96 & 0.97 & 0.96 \\
\hline 22 & 29.3 & 20.2 & 1.00 & 0.99 & 1.00 & 0.96 & 1.00 & 0.98 \\
\hline 23 & 9.9 & 24.0 & 1.00 & 0.99 & 1.00 & 0.99 & 1.00 & 0.99 \\
\hline Mean & $18.7^{*}$ & 14.4 & 0.997 & 0.996 & 0.996 & 0.991 & 0.996 & 0.993 \\
\hline SD & 7.8 & 4.7 & 0.008 & 0.010 & 0.009 & 0.011 & 0.008 & 0.010 \\
\hline
\end{tabular}

(subject 17). Compared with literature [11, 12, 18-20], accuracy outcomes reported here are very encouraging.

The accurate classification ability and the efficient post-processing of model output guaranteed mean prediction, recall, and F1-score values in gait-event assessment very close to 1 . Moreover, an average $M A E$ over population of $14.4 \pm 4.7 \mathrm{~ms}$ (Table 2) and $23.7 \pm 11.3 \mathrm{~ms}$ (Table 3 ) was detected in the prediction of HS and TO, respectively. To appreciate the quality of these predictions, it is helpful highlighting that associated MAEs correspond approximately to $1 \%$ and $2 \%$ of a gait cycle duration, respectively. Since large variability of the signal to predict (foot-floor contact) is expected to affect the performance of a classifier, an added value is that present results have been achieved in condition of high variability of foot-switch signals. In fact, the eight-shaped path followed by subjects during the experimental procedure is acknowledged to introduce further gait variability, due to curves, reversing, deceleration, and acceleration, with respect to straight or treadmill walking. To the best of our knowledge, only two studies on this issue are available and neither reported such a small prediction error [11, 12]. Moreover, these outcomes are at least comparable with those reported by analogous IMU studies. Very meaningful from this point of view is the study where 17 IMU-based approaches 
Table 3 MAE (mean absolute error), precision, recall, and F1-score provided by inter and intra-subject approach for Toe-Off (TO) prediction

\begin{tabular}{|c|c|c|c|c|c|c|c|c|}
\hline \multirow{2}{*}{$\begin{array}{l}\text { TO } \\
\text { Subject }\end{array}$} & \multicolumn{2}{|c|}{ MAE (ms) } & \multicolumn{2}{|c|}{ Precision } & \multicolumn{2}{|l|}{ Recall } & \multicolumn{2}{|c|}{ F1-score } \\
\hline & Inter & Intra & Inter & Intra & Inter & Intra & Inter & Intra \\
\hline 1 & 12.3 & 14.1 & 1.00 & 0.99 & 1.00 & 1.00 & 1.00 & 1.00 \\
\hline 2 & 25.8 & 21.8 & 0.97 & 1.00 & 0.98 & 0.98 & 0.97 & 0.99 \\
\hline 3 & 27.6 & 27.6 & 0.98 & 0.98 & 1.00 & 0.98 & 0.99 & 0.98 \\
\hline 4 & 104.2 & 49.7 & 1.00 & 1.00 & 0.99 & 0.98 & 0.99 & 0.99 \\
\hline 5 & 36.0 & 16.4 & 1.00 & 1.00 & 1.00 & 0.99 & 1.00 & 0.99 \\
\hline 6 & 44.7 & 25.3 & 1.00 & 1.00 & 1.00 & 0.98 & 1.00 & 0.99 \\
\hline 7 & 37.6 & 13.3 & 1.00 & 1.00 & 1.00 & 0.98 & 1.00 & 0.99 \\
\hline 8 & 27.9 & 12.0 & 1.00 & 1.00 & 1.00 & 0.99 & 1.00 & 0.99 \\
\hline 9 & 45.1 & 28.4 & 1.00 & 1.00 & 1.00 & 0.99 & 1.00 & 0.99 \\
\hline 10 & 53.4 & 13.3 & 1.00 & 1.00 & 1.00 & 0.98 & 1.00 & 0.99 \\
\hline 11 & 55.6 & 52.9 & 0.94 & 0.96 & 0.93 & 0.93 & 0.93 & 0.95 \\
\hline 12 & 65.8 & 29.6 & 1.00 & 0.99 & 1.00 & 0.98 & 1.00 & 0.98 \\
\hline 13 & 24.1 & 15.5 & 0.98 & 1.00 & 0.96 & 1.00 & 0.97 & 1.00 \\
\hline 14 & 29.4 & 19.6 & 1.00 & 1.00 & 1.00 & 1.00 & 1.00 & 1.00 \\
\hline 15 & 40.3 & 21.1 & 1.00 & 1.00 & 1.00 & 1.00 & 1.00 & 1.00 \\
\hline 16 & 22.7 & 17.2 & 1.00 & 1.00 & 1.00 & 1.00 & 1.00 & 1.00 \\
\hline 17 & 28.9 & 11.9 & 1.00 & 1.00 & 1.00 & 1.00 & 1.00 & 1.00 \\
\hline 18 & 12.4 & 14.1 & 1.00 & 0.99 & 1.00 & 1.00 & 1.00 & 1.00 \\
\hline 19 & 15.7 & 21.8 & 1.00 & 1.00 & 1.00 & 0.98 & 1.00 & 0.99 \\
\hline 20 & 15.9 & 27.6 & 1.00 & 0.98 & 1.00 & 0.98 & 1.00 & 0.98 \\
\hline 21 & 38.1 & 39.7 & 0.97 & 0.92 & 0.96 & 0.92 & 0.96 & 0.92 \\
\hline 22 & 32.6 & 30.0 & 0.98 & 0.99 & 0.98 & 0.96 & 0.98 & 0.98 \\
\hline 23 & 12.1 & 23.0 & 1.00 & 0.99 & 1.00 & 0.98 & 1.00 & 0.98 \\
\hline Mean & $35.1^{*}$ & 23.7 & 0.991 & 0.990 & 0.990 & 0.980 & 0.991 & 0.985 \\
\hline SD & 20.9 & 11.3 & 0.008 & 0.018 & 0.019 & 0.020 & 0.017 & 0.018 \\
\hline
\end{tabular}

were tested on 35 healthy subjects [1], showing a media time error ranging from 60 to $65 \mathrm{~ms}$ and from -25 to $6 \mathrm{~ms}$ for HS and TO prediction, respectively, and a 25th-75th percentile error ranging from 40 to $111 \mathrm{~ms}$ and from 68 to $120 \mathrm{~ms}$ for HS and TO prediction, respectively. A very recent study remarks the same perspective [23], reporting a 17.83-ms HS-MAE and a 26.96-ms TO-MAE during indoor walking. Thus, we may argue that in specific environments, EMG-based approach may be very useful and as much accurate as other techniques.

These promising performances are supposed to be likely due to the peculiarities of the experimental protocol which provides a great amount of data per subject: numerous strides (about 500) and ten different sEMG signals (five muscles per leg). The choice of the model was made based on the analysis we carried out in $[12,18]$. The first study [12] compared the classification accuracy on inter-subject data of five different MLP models of increasing complexity in terms of classification. The second study [18] compared the classification accuracy on intra-subject data of the first three MLP models introduced in [12]. In both studies, the neural network with three hidden layers composed of 512, 256 and 128 units was identified as the model achieving the best accuracy on unlearned subjects. That is the model used in the present study. Trying to reduce the complexity of the 
neural network, an MLP model with a single hidden layer was tested on the intra-subject data. The number of nodes was set at a value (128) lower than the number of inputs (200). Results show that the single-layer model achieves a mean classification accuracy on test set of $95.7 \pm 1.6 \%$ vs. $96.1 \pm 1.9 \%$ reported by the chosen model, confirming that also in the present population the 3-hidden-layer model allows obtaining (slightly) better performances.

The robustness of MLP models for gait-phase classification has been previously validated for the inter-subject approach [12]. Thus, the present intra-subject approach was tested by a direct comparison with the performances achieved by the inter-subject one with the same model in the same population. It is worth noticing that, even if in the present study the neural network is the same used in [12], results in both intra-subject and inter-subject are completely new because (1) the training phase was performed including ten sEMG signals (i.e., 5 muscles per leg) vs. the eight sEMG signal used in [12], where RFs were not included; (2) to our knowledge this is the first attempt to provide HS and TO estimation by means of an intra-subject approach. Moreover, the inter-subject approach implemented in the present study improves already the performances reported in [12]. Comparison between intra-subject and the inter-subject approaches (Tables 2 and 3) highlights two main points: the intra-subject approach allows to achieve (1) an improvement of $1.4 \%$ of mean classification accuracy (96.1\% vs. $94.7 \%$ ); and (2) a significant $(p<0.05)$ decrease of mean MAE in the prediction of both HS (23\% reduction) and TO (33\% reduction) timing. Despite this, on average also the inter-subject approach appears to work adequately, especially for HS. However, mean value over population often disguises what really happen in a single specific subject. The reason why a different way was tried, introducing the intra-subject approach, is that sometimes HS and, above all, TO predictions in a single subject are not so accurate with the inter-subject approach. To stress this fact, Fig. 2 has been introduced, comparing the prediction in each single subject provided by the two approaches (inter-subject in red and intra-subject in cyan). Yellow arrows in Fig. 2 indicate inter-subject predictions which diverge more evidently from the mean values (18.7 ms for HS and 35.1 for TO). Some of these outliers present very bad prediction (S17 for HS and S4 for TO above all). This problem does not occur for intra-subject predictions, which spread out around the mean value showing a small dispersion of MAE values. This consideration becomes clearer if the single case is discussed. As clearly highlighted in Fig. 2, subject 4 (S4 yellow arrow in panel B) shows very bad inter-subject TO prediction, where an MAE of $104.2 \mathrm{~ms}$ is detected. From the clinical point of view such an error is not acceptable because $104.2 \mathrm{~ms}$ is about $10 \%$ of gait cycle. Thus, we can say that intra-subject approach fails in the prediction of TO in subject 4 . Also for intra-subject approach, TO in subject 4 is one of the hardest to predict, but the MAE remains $<50 \mathrm{~ms}$. In this case, there is $54.5 \mathrm{~ms}$ difference between the two approaches (nearly $50 \%$ reduction of absolute error). The intra-subject approach has been introduced just to fix situation like this. Similar condition has been detected also in subject 12 ( $\mathrm{S} 12$ yellow arrow in panel $\mathrm{B}, \mathrm{TO}-\mathrm{MAE}=65.8 \mathrm{~ms}$ for inter-subject approach and $\mathrm{TO}-\mathrm{MAE}=29.6 \mathrm{~ms}$ for intra-subject approach, difference $=36.2 \mathrm{~ms}, 45 \%$ reduction of absolute error). Inter-subject HS prediction reports the same problem. Subject 17 (S17 yellow arrow in panel A) shows the worst HS prediction (HS-MAE $=36.5 \mathrm{~ms}$ ) for intersubject approach. Intra-subject approach reduces MAE to $9.2 \mathrm{~ms}$ (difference $=27.3 \mathrm{~ms}$, 

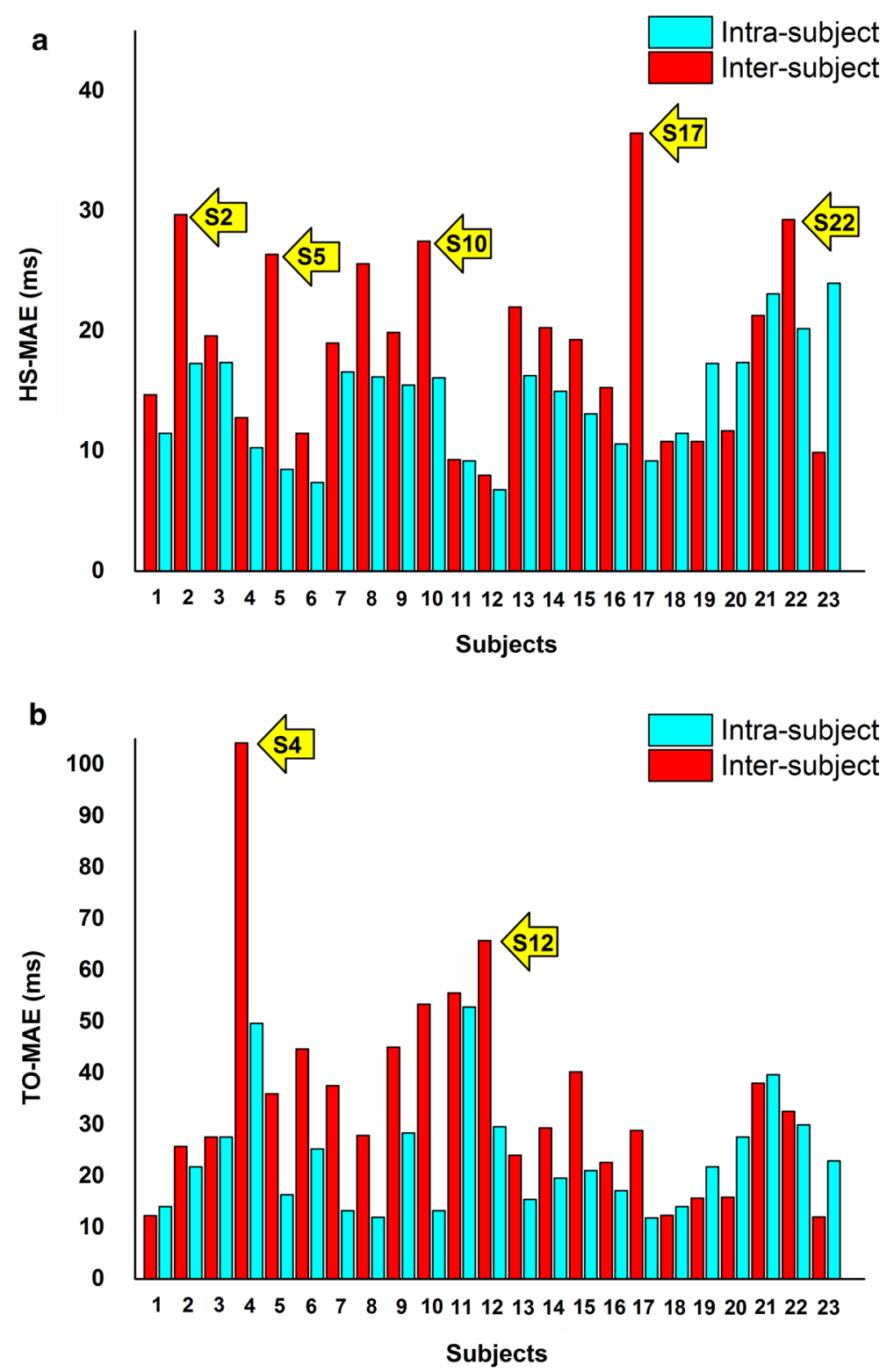

Fig. 2 Prediction of HS-MAE (a) and TO-MAE (b) in each single subject provided by inter-subject (red bars) and intra-subject approaches (cyan bars). Yellow arrows indicate inter-subject predictions which diverge more evidently from the mean values

$75 \%$ reduction of absolute error). The same goes for S2, S5, S10, and S22. In all these cases (at least 7 out of 23), the inter-subject approach seems not to work adequately and the contribution of the intra-subject approach appears to be very valuable.

Cited reports $[11,12]$ identified more difficulties in detecting toe-offs rather than heelstrikes. Accelerometer-based studies provided the same indication [21]. The inter-subject approach computed in the present study confirms this trend, showing $M A E$ value in TO prediction up to $104 \mathrm{~ms}$. The proposed intra-subject approach also shows better performance in detecting heel-strikes rather than toe-offs, but with the advantage of limiting the worst-case $M A E$ (subject 11) below $53 \mathrm{~ms}$. Ultimately, all these outcomes 
work together to argue that intra-subject approach outperforms the inter-subject one for this specific task. Proving this is the actual added value of the present study.

In Tables 2 and 3, a time tolerance T of $600 \mathrm{~ms}$ is employed to predict HS and TO (as discussed in "Gait-event identification" section). To provide an indirect comparison with previous studies where different approaches based on kinematic data were presented, an additional evaluation is provided. Following the evaluation approach adopted in [22] for IMU-based prediction, the time tolerance $\mathrm{T}$ was set to $50 \mathrm{~ms}$ (100 samples), which corresponds to the typical error reported in literature [24]. Average results over the 23 subjects are reported in Table 4, for both HS and TO predictions. As expected, reducing $\mathrm{T}$ to $50 \mathrm{~ms}$ implies a worsening of precision and recall, but concomitantly it improves $M A E$ values, for both HS and TO estimation. However, such results confirm that performances provided by the intra-subject approach are sensibly better than those achieved with the inter-subject approach, both in F1-score and in MAE. Furthermore, the percentage of HS detected within $50 \mathrm{~ms}$ is still relatively large $(95 \%$ and $93 \%$ for intrasubject and inter-subject approaches, respectively), with an average $M A E$ of $13.2 \mathrm{~ms}$ (inter-subject) and $10.9 \mathrm{~ms}$ (intra-subject). TO predictions are, in general, less accurate. However, when using the intra-subject approach, around $90 \%$ are detected within $50 \mathrm{~ms}$ with an average $M A E$ of $14.6 \mathrm{~ms}$. These results are comparable with those reported for IMU studies [22]. In the present paper, signal windows of $10 \mathrm{~ms}$ are considered to feed the neural network in both intra- and inter-subject approaches, according to [12]. Further investigation of parameter tuning (involving signal segmentation and windowing) could be an interesting future direction.

As introduced above, the clinically useful purpose of the study is the attempt at reducing the complexity of the experimental gait analysis set-up, predicting the foot-floorcontact data from neural network interpretation of EMG signal in a single specific subject. The fewer sensors are used, the easier is to safeguard patient comfort. This is true for both inter-subject and intra-subject approaches. To run the inter-subject approach, a large dataset of EMG and foot-floor-contact signals from many different subjects is needed to train the neural network [12]. When such a dataset is available, classification of a new subject does not require further training (and the use of further sensors, such as IMUs or foot-switch sensors) and the inter-subject approach is preferable. However, this is true for those populations where large amounts of data are available. On the contrary, recruiting an adequate number of subjects to build up the dataset for specific condition, pathology, or dysfunction could be a challenging task. This is particularly true for those disorders which are uncommon or rare. The intra-subject approach has been introduced

Table 4 MAE (mean absolute error), precision, recall, and F1-score provided by inter and intra-subject approach using a time tolerance $\boldsymbol{T}=\mathbf{5 0} \mathbf{~ m s}$

\begin{tabular}{|c|c|c|c|c|c|c|c|c|}
\hline \multirow[t]{2}{*}{ Subject } & \multicolumn{2}{|c|}{ MAE (ms) } & \multicolumn{2}{|c|}{ Precision } & \multicolumn{2}{|c|}{ Recall } & \multicolumn{2}{|c|}{ F1-score } \\
\hline & Inter & Intra & Inter & Intra & Inter & Intra & Inter & Intra \\
\hline HS mean & 13.2 & 10.9 & 0.93 & 0.95 & 0.92 & 0.95 & 0.93 & 0.95 \\
\hline SD & 5.6 & 2.5 & 0.06 & 0.04 & 0.06 & 0.04 & 0.06 & 0.04 \\
\hline TO mean & 21.8 & 14.6 & 0.79 & 0.89 & 0.78 & 0.88 & 0.78 & 0.89 \\
\hline SD & 8.2 & 4.0 & 0.20 & 0.10 & 0.20 & 0.11 & 0.20 & 0.10 \\
\hline
\end{tabular}


also to provide an alternative way to face this experimental issue. In this approach, only data from the single subject under examination are necessary. It is true that the neural network should be trained with EMG and basographic data for each new subject, but only once. For all the successive tests, no further training is required and further sensors are not necessary. It is acknowledged that for monitoring their rehabilitation process, different kind of patients (neurological disorders, injuries, aging) should undergo periodical tests. The intra-subject approach appears to be very suitable for these cases, since after a first session where EMG and basographic data have to be acquired and the model has to be trained, all the following tests do not need neither the basographic signal acquisition nor the training of the model, but only the acquisition of EMG signals of the single patient under examination. Moreover, the higher precision in gait-event prediction provided by the intra-subject approach allows to correctly detect the small improvements of the patient performances in temporal parameters during rehabilitation and to handle data where duration of gait phases could be strongly altered (aging, Parkinson's disease) $[25,26]$. In these conditions, the intra-subject approach is probably preferable. In conclusion, intra-subject approach has its pros and cons, but its real added value is that it works just when the inter-subject approach seems to fail, because of bad individual performances or the lack of a large dataset of EMG and foot-floor-contact signals to train the neural network.

The present intra-subject approach could also play a relevant role in the so-called Statistical Gait Analysis (SGA). SGA is a recently developed technique, based on the cycle-dependency of muscular activation, which has already provided promising results published in many studies of the present $[13,27]$ and other groups of researchers $[28$, 29]. SGA allows to characterize human walking by means of average sEMG features and spatial-temporal parameters extracted from hundreds of consecutive strides per trial that are recorded and classified according to the sequence of foot-floor contact. Intrasubject approach could contribute in reducing the number of instrumentation worn by the subject and necessary for SGA, removing the need of foot-switches. Further fields of application could be exoskeletons [30], smart prostheses, [31] and functional electronic simulation [32].

From the methodological point of view, future developments of the present study could focus on testing the classification/prediction performance provided by a mixed approach. It could consist in testing the neural network in brand new strides of a subject, after training the neural network with EMG data measured in a population of homogeneous subjects (i.e., with similar characteristics, such as age, weight, and height) which includes also the subject under investigation. For example, the neural network could be trained with the $100 \%$ of the signals acquired in 22 subjects and the $90 \%$ of the signal acquired in a 23rd subject. Then, the neural network could be tested in the remaining $10 \%$ of the signal acquired in the 23 rd subject.

For clinical and research purposes, stance phase is typically split in three main subphases: heel-strike, flat foot contact, and push off. A further advancement could involve the attempt of classifying stance sub-phases and predicting transition timing between them. Moreover, present results are achieved from data acquired during walking at selfselected speed. The potential effect of gait speed could be also considered, as reported 
in [22]. This would also be an interesting future direction for the present work, as EMG envelopes show adaptations to different gait speeds.

\section{Conclusions}

The present study proposes an accurate methodology for classifying stance vs. swing and predicting the timing of gait events, based on neural network interpretation of intrasubject sEMG data during walking. The clinically useful contribution of the study consists in predicting gait events from only EMG signals, contributing to remove the need of further sensors for the direct measurement of temporal data. A direct comparison in the same population showed that results of the present approach outperform the outcomes provided by the same network with an inter-subject approach. However, the choice of the suitable approach should be driven not only by network performance but also (mainly) by patient comfort and clinical needs.

\section{Methodology}

\section{Subjects}

The dataset included signals recorded from 23 healthy adults (12 females and 11 males). Mean $( \pm S D)$ characteristics were age $=23.8 \pm 1.9$ years; height $=173 \pm 10 \mathrm{~cm}$; mass $=63.3 \pm 12.4 \mathrm{~kg}$. None of the subjects presented any pathological condition or had undergone orthopedic surgery that might have affected lower limb mechanics. Therefore, subjects with joint pain, neurological pathologies, orthopedic surgery, and abnormal gait were not recruited. Overweight and obese subjects (body mass index $>25$ ) were not recruited because obesity is acknowledged to affect muscle function during walking [33].

\section{Signal acquisition}

The multichannel recording system Step32 (Medical Technology, Italy, Version PCI-32 ch2.0.1. DV; resolution: 12 bit; sampling rate: $2 \mathrm{kHz}$ ) was employed for the acquisition of all signals. The use of a single system guaranteed the synchronization of all signals. Participants have been instrumented with five sEMG probes and three foot-switches for each leg. Then, they walked with bare feet at self-selected pace (i.e., their own usual, comfortable pace) following an eight-shaped path (Fig. 3), which includes natural deceleration, reversing, curve and acceleration, in the Motion Analysis Lab (Università Politecnica delle Marche, Ancona, Italy), for around 5 min. sEMG signals were registered by means of three single-differential probes with fixed geometry (fixed inter-electrode distance of $8 \mathrm{~mm}$ ) placed over tibialis anterior (TA), gastrocnemius lateralis (GL), and medial hamstrings $(\mathrm{MH})$ and two single-differential probes with variable geometry (variable inter-electrode distance, starting from a minimum of $12 \mathrm{~mm}$ ) placed over vastus lateralis $(\mathrm{VL})$ and rectus femoris $(\mathrm{RF})$. Electrode location and orientation were performed under the supervision of a skilled licensed physical therapist, following the SENIAM recommendations [34]. Foot-switches were attached under the heel, the first and the fifth metatarsal heads of each foot for measuring foot-floor-contact signal [27]. Experimental set-up is depicted in Fig. 4. Further details about signal acquisition procedure can be found in [13]. 


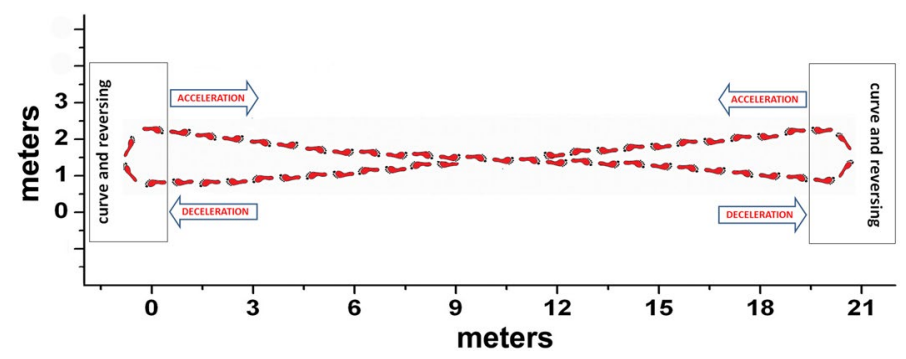

Fig. 3 Eight-shaped trajectory followed by each subject during walking

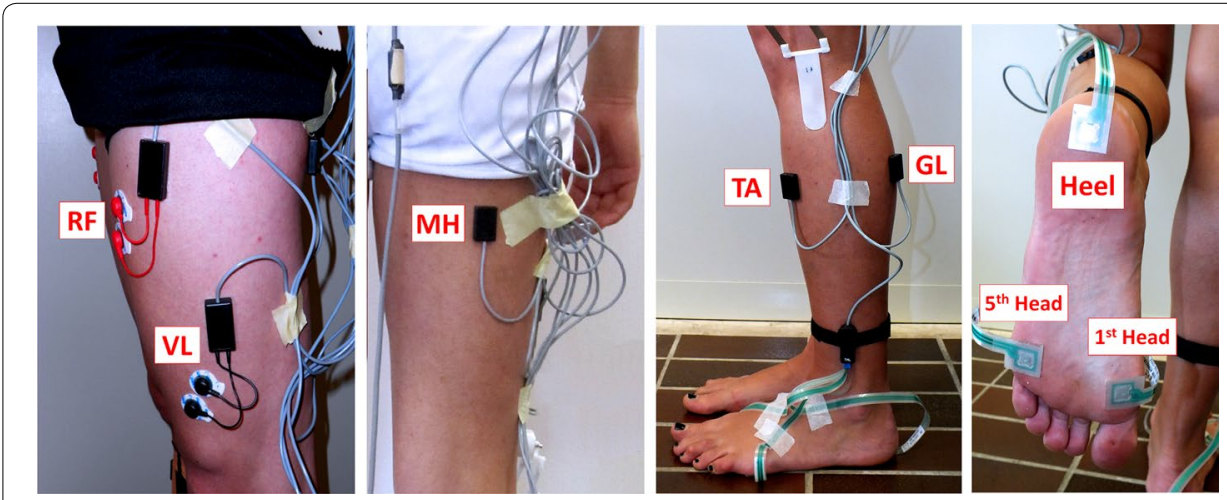

Fig. 4 Experimental set-up. sEMG probes are applied over rectus femoris (RF), vastus lateralis (VL), medial hamstrings $(\mathrm{MH})$ tibialis anterior (TA), and gastrocnemius lateralis (GL). Foot-switches are attached under the heel, the first (1st Head) and the fifth (5th Head) metatarsal heads

\section{Signal pre-processing}

sEMG signals were amplified, high-pass filtered (linear-phase FIR filter, cut-off frequency: $20 \mathrm{~Hz}$ ) and low-pass filtered (linear-phase FIR filter, cut-off frequency: $450 \mathrm{~Hz}$ ) for removing motion artifact and high-frequency noise, respectively. Then, the envelope of signal was extracted after a full-wave rectification, applying second-order Butterworth low-pass filter following the classic indication provided by acknowledged studies by Winter et al. [35, 36] and Hermens et al. [34]. Winter proposed a cut-off frequency of $3 \mathrm{~Hz}[35,36]$, while Hermens suggested a cut-off frequency of $10 \mathrm{~Hz}$ [34]. In the present paper, a cut-off frequency of $5 \mathrm{~Hz}$ was adopted, as a good compromise between the two approaches. Zero-phase digital filtering was performed to avoid phase shift. Eventually, each sEMG signal was min-max normalized within each subject and for each muscle, thus mapping the values in the [0-1] interval. An example of normalized envelopes in a representative stride is reported in Fig. 5. Foot-switch signals were processed for identifying the different gait cycles and phases (stance and swing), according to the approach discussed in [37]. The study [37] describes and validates an algorithm for the segmentation into separate gait cycles of the basographic signal, measured by means of three foot-switches during walking. The algorithm is able to provide also the four main gait sub-phases: heel contact $(\mathrm{H})$, flat foot contact $(\mathrm{F})$, push off or heel off $(\mathrm{P})$, swing $(\mathrm{S})$. Based on this segmentation, the algorithm classifies the different gait cycles characterized by a specific sequence of gait phases (HFPS, PFPS, FPS and so on). 

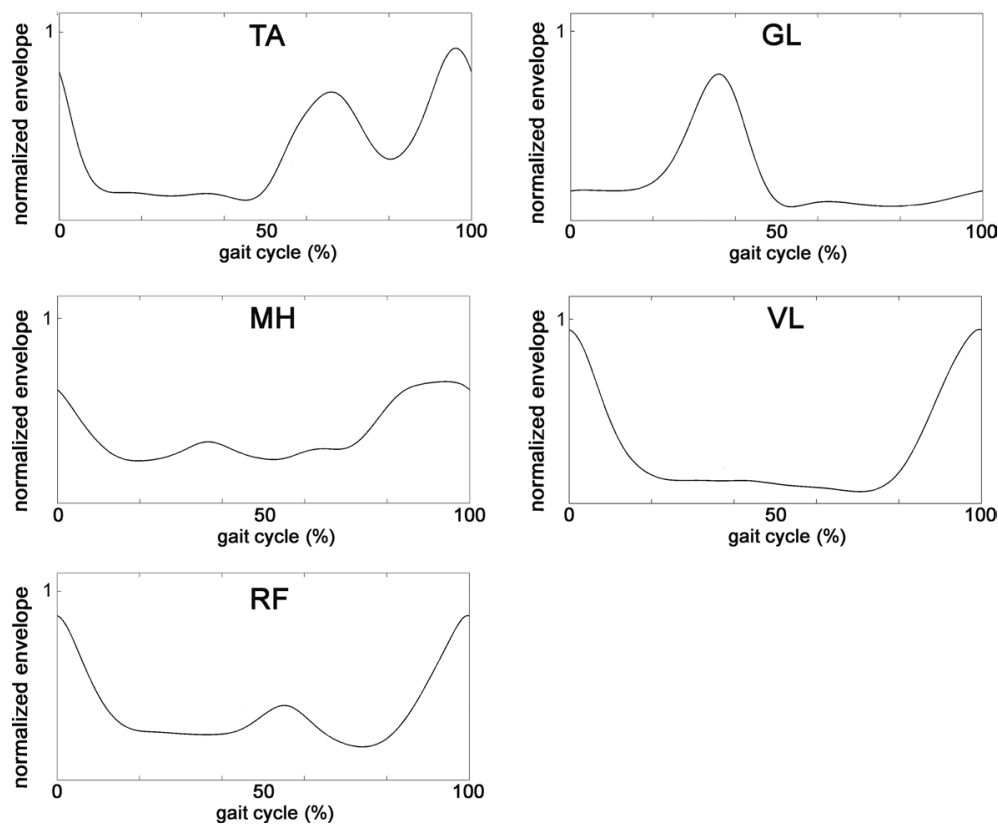

Fig. 5 The envelope in a representative stride resulting from the pre-processing of the raw sEMG signals recorded from the five muscles of the right leg

\section{Data preparation}

In our experiments, the neural network classifier is fed with the envelope of the EMG signals, thus the classification is based on hidden features automatically learned from the network. As demonstrated in [12], this approach provides better performances than using hand-crafted time-dependent features. For suitably training the classifier, each sEMG signal was split into 20 -sample windows (corresponding to $10 \mathrm{~ms}$ ). Examples of 20-sample windows taken from two consecutive steps of a representative subject are depicted in Fig. 6. A chronological sequence of 200-sample vectors was created, where each vector included the ten synchronized 20-sample windows from the sEMG signals of the ten muscles (five for each leg). In details, the first sample of the first 200-sample vector of the sequence was the first sample of the EMG signal from the muscle 1 (TA, right leg), the second sample of the first 200-sample vector was the first sample of the EMG signal from the muscle 2 (GL, right leg), and so on up to the tenth muscle (RF, left leg). Then, a specific label was assigned to each 200-sample vector as follows: if the value of all the samples of the basographic signal corresponding to the 200-sample vector was 0 (or 1), a global label 0 (or 1 ) was assigned to the 200 -sample vector. 200 -sample vectors including swing-to-stance or stance-to-swing transitions were not considered during phase classification, as the goal was to train the network to distinguish between stance and swing phases. On the contrary, all the windows, including those corresponding to a phase transition, were used to predict the timing of gait events (see "Gait-event identification" section).

\section{Training the classifier}

Two different approaches for training the classifier were tested and compared: the intrasubject (Fig. 7) and the inter-subject approach (Fig. 8). 


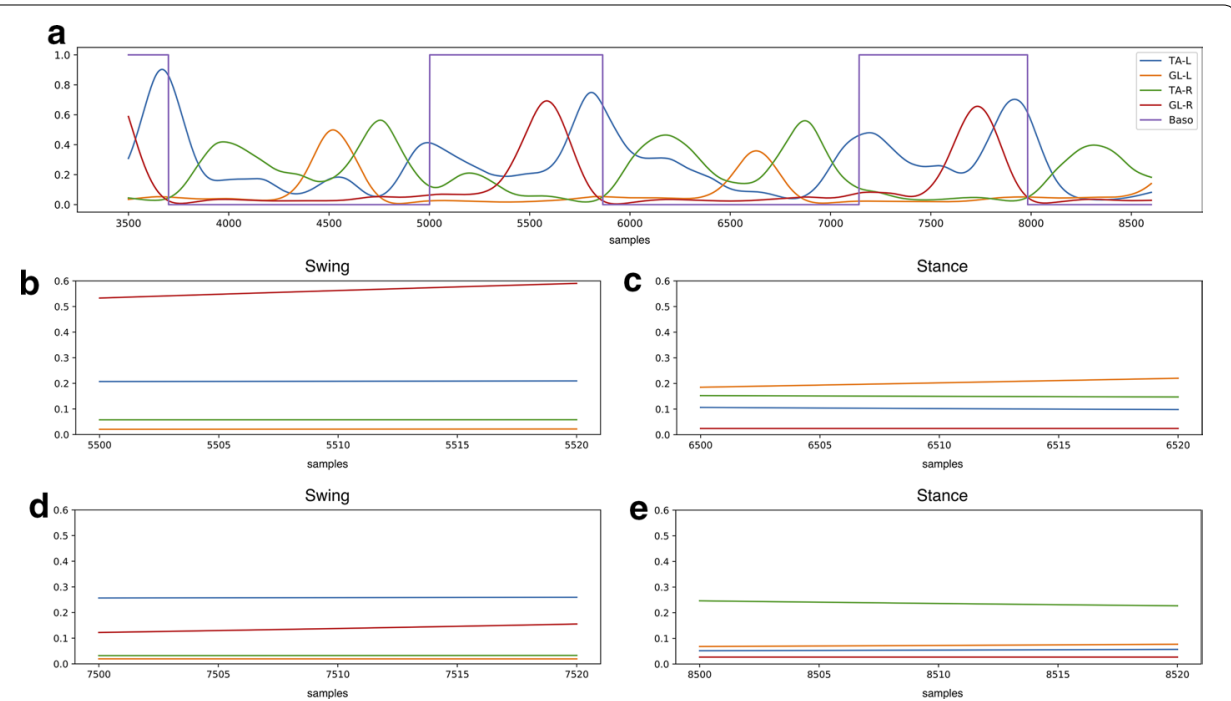

Fig. 6 a Example of a normalized full-wave rectified and enveloped signal from left (blue line) and right (green line) tibialis anterior and left (orange line) and right (brown line) gastrocnemius lateralis, superimposed to left basographic signal (violet line), in two strides of a representative subject. $\mathbf{b}$, d Two representative 20-sample windows taken from the swing phases of the signal depicted in $\mathbf{a}$. c, e Two representative 20-sample windows taken from the stance phases of the signal depicted in panel a)

\section{Intra-subject approach}

The approach is based on the attempt at training the Neural Network classifier by means of sEMG data from a single subject and then classifying gait phases in unseen stride sequences of the same subject. To this aim, the signal composed by the chronological sequence of 200-sample vectors characterizing the walking of a single subject was processed as follows: it was split into 10 equal slots of approximately 60,000 samples long each (Fig. 7). Nine out of ten slots were used to train the classifier. All the 200-sample vectors picked up from the nine training slots were provided to the neural network for the training phase. The 200-sample vectors from the remaining single slot were used for the testing phase, considering the corresponding foot-switch signal as ground truth. The procedure was performed ten times, each time using a different slot as test set (tenfold cross-validation). Classification results in each subject were provided as mean value ( \pm standard deviation, SD) over the tenfold. Population (global) results were provided as mean value $( \pm \mathrm{SD})$ over the 23 subjects.

\section{Inter-subject approach}

The approach is based on the attempt at training the Neural Network classifier by means of sEMG data from 22 subjects (out of 23 subjects of the present population) and then classifying gait phases in the remaining unseen subject (leave-one-out cross-validation procedure). To this aim, all the 200-sample vectors were picked up from the signals of the 22 subjects and then provided as input to the neural network for the training phase. The 200-sample vectors from the remaining single subject were used for the testing phase, considering the corresponding foot-switch signal as ground truth. The procedure was performed 23 times, each time using a different subject as test set (23-fold crossvalidation). Results in each subject were provided as the classification results in a single 


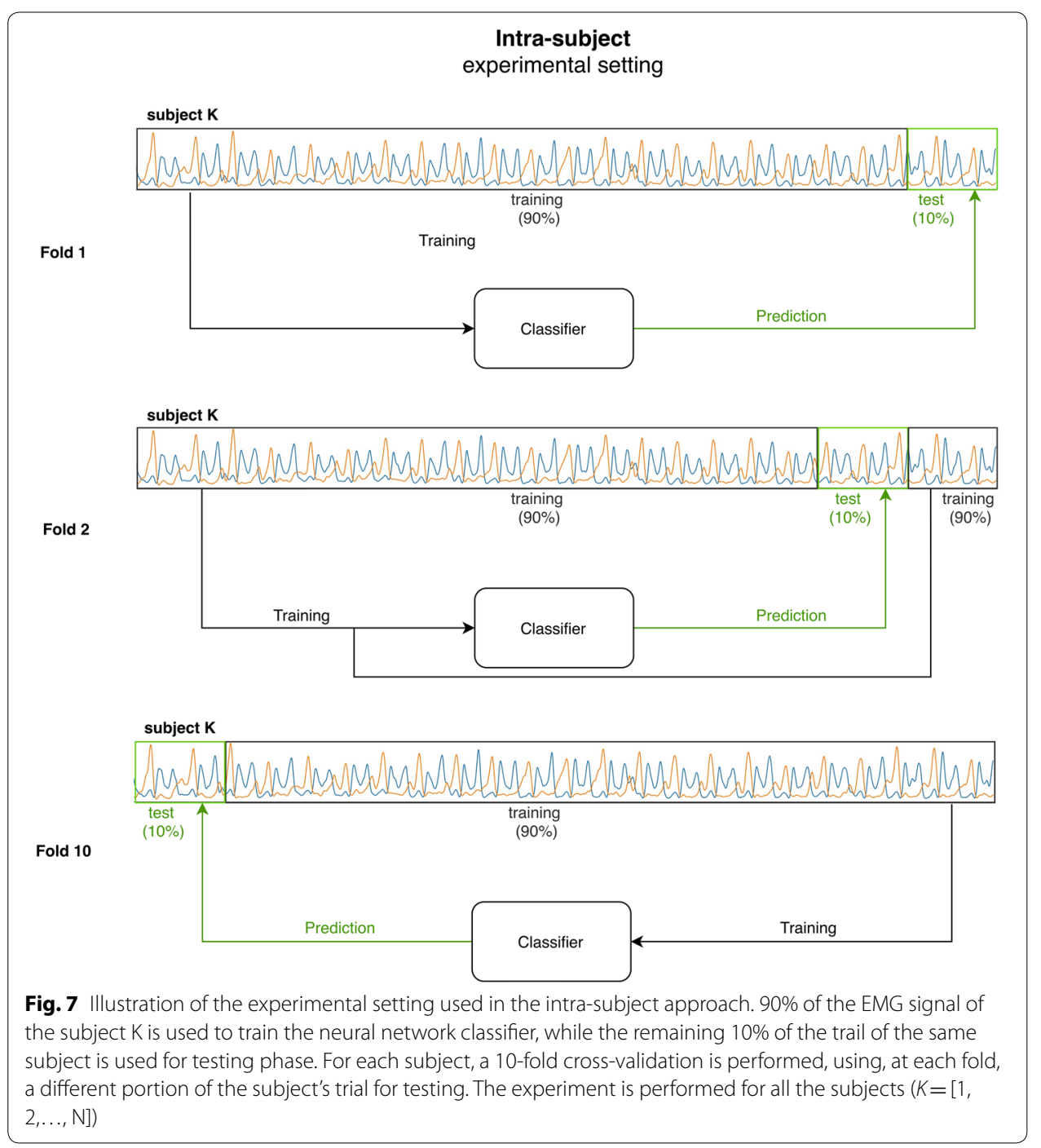

fold. Population (global) results were provided as mean value $( \pm S D)$ over the 23-fold. Further details about this approach can be found in [12].

\section{The neural network}

Multi-layer perceptron (MLP) architecture was implemented in the present study. The model was a neural network with one input layer composed of 200 units (corresponding to the 200 signal values forming each window), three hidden layers composed of 512, 256 and 128 neurons, and a one-dimensional output. The output was fed to a sigmoid function and a 0.5 threshold was used to achieve a binary output: when the output of the sigmoid was $>0.5$ the label 1 was assigned, otherwise the label 0 was assigned. Rectified linear units (ReLU) were implemented to provide non-linearity between two consecutive hidden layers. In the experiments, stochastic gradient descent was employed as the optimization algorithm and binary cross-entropy as the loss function. Learning rate was experimentally set to 0.01 . Eventually, MLP model was trained adopting an early stop technique: the network was trained for a maximum of 100 epochs, stopping when 


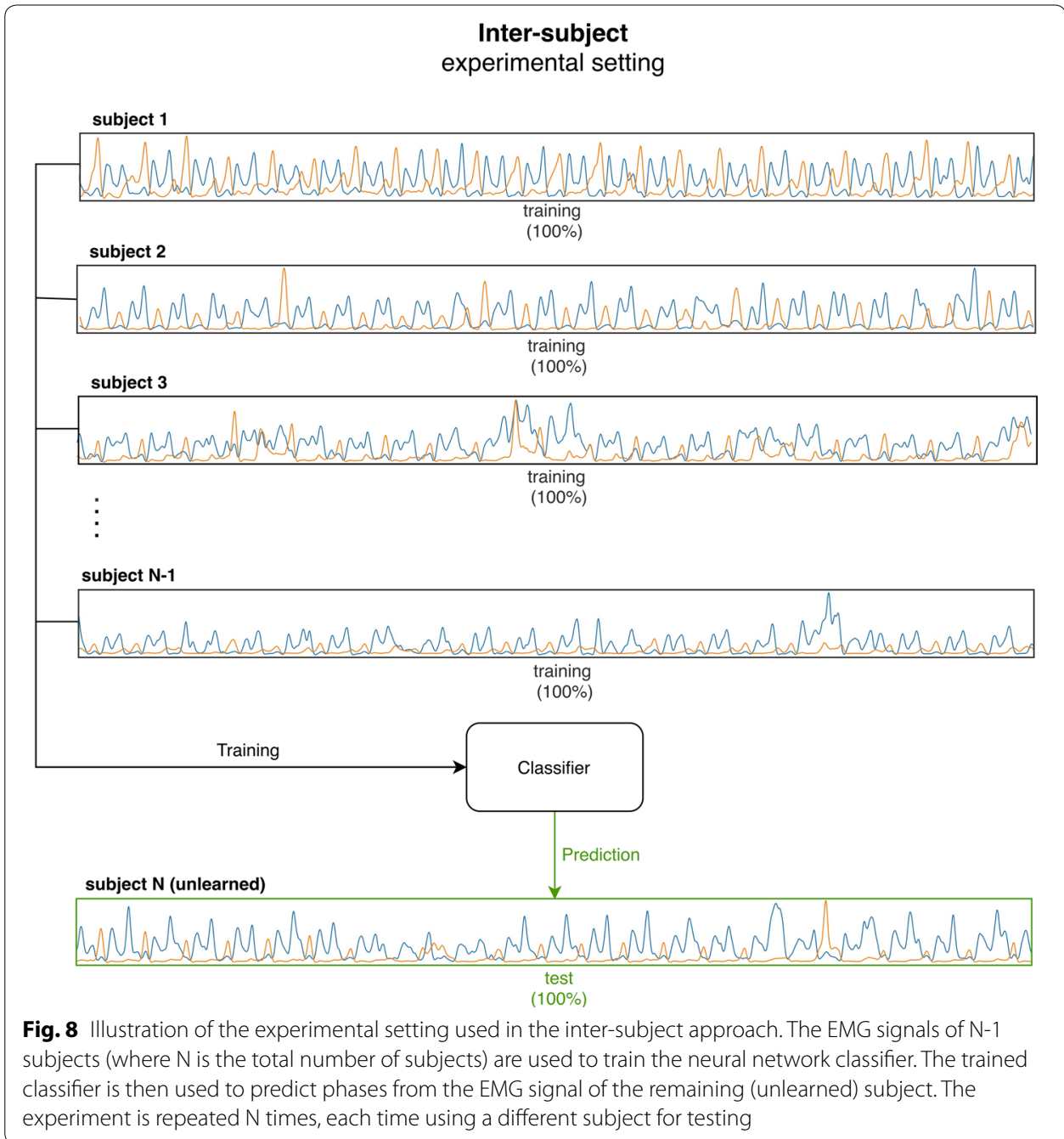

the accuracy on the validation set (composed of the last 10\% of subject strides) did not increase for 10 consecutive epochs. We stored the network weights learned at the epoch providing the best accuracy on the validation set and then used such a trained model to evaluate performances on the test sets.

\section{Gait-event identification}

The foot-floor-contact signal was predicted by chronologically arranging the binary output of MLP network. A vector was provided as output, where sequences of 1 (swing phase) alternate with sequences of 0 (stance phase). Literature reported that stance and swing phase during healthy walking at typical speed last on average $60 \%$ and $40 \%$ of gait cycle [38]. Starting from this observation, all the transitions in the foot-floor-contact signal lasting less than 175 ms were rejected ( $\approx 16 \%$ of gait cycle). During the post-processing of the predicted basographic signal, 180 spikes (false phases) per subject were removed on average. Average spike duration was $7.8 \pm 32.8$ samples, corresponding to $3.9 \pm 16.4 \mathrm{~ms}$. Then, gait events were identified in the 
cleaned signal. Swing-to-stance transitions (heel-strike, HS) were assessed as the sample when the sample value switched from 1 to 0 . In the same way, stance-to-swing transitions (toe-off, TO) were assessed as the sample when the sample value switched from 0 to 1. Performance of predictions was provided in terms of precision, recall, and F1-score. Precision is defined as

$$
\text { Precision }=\frac{T P}{T P+F P}
$$

where $T P$ s are true positives and $F P$ s are false positives. Recall is defined as

$$
\text { Recall }=\frac{T P}{T P+F N}
$$

where $F N$ s are false negatives. F1-score is defined as

$$
F 1-\text { score }=2 \cdot \frac{\text { Precision } \cdot \text { Recall }}{\text { Precision }+ \text { Recall }}
$$

A predicted HS or TO at time $t_{p}$ was acknowledged as true positive (TP) if an event of the same type occurs in the ground truth signal at time $t_{g}$ such that $\left|t_{g}-t_{p}\right|<\mathrm{T}$. T is a temporal tolerance, set to $600 \mathrm{~ms}$. Otherwise, the predicted event was acknowledged as a false positive $(F P)$. For all the true positives, mean absolute error $(M A E)$ was computed as the average time distance between the predicted event and the corresponding one in ground truth signal.

\section{Statistics}

To test the significance of the difference of data distributions (MAE, precision, recall, F1-score) between intra-subject and inter-subject approaches, statistical tests were performed. First of all, the normality of each data distribution was evaluated by means of Shapiro-Wilk test. Then, two-tailed, non-paired Student's $t$ test was performed to evaluate the significance of the difference between normally distributed samples. In the same way, Kruskal-Wallis test was performed to evaluate the significance of the difference between non-normally distributed samples. Statistical significance for each test was set at $5 \%$.

\section{Abbreviations}

EMG: Electromyography; FN: False negative; FP: False positive; GL: Gastrocnemius lateralis; HS: Heel-strike; IMUs: Inertial measurement units; MAE: Mean absolute error; MH: Medial hamstrings; MLP: Multi-layer perceptron; ReLU: Rectified linear units; RF: Rectus femoris; sEMG: Surface electromyography; TA: Tibialis anterior; TO: Toe-off; TP: True positive; VL: Vastus lateralis.

Acknowledgements

Not applicable. The present paper was awarded the "SIAMOC Best Methodological Paper 2019".

Authors' contributions

Conceptualization: FDN, CM, SF; methodology: FDN, CM; software: CM, GM investigation: CM, FDN; data curation: FDN, CM, GM, FV; writing — original draft preparation: FDN; writing — review and editing, CM, FV, SF; supervision SF. All authors read and approved the final manuscript.

\section{Funding}

No funding was received. 
Availability of data and materials

SEMG and basographic data analyzed in the present study are going to be published in a public dataset, and are currently available for research purposes by contacting the authors.

\section{Ethics approval and consent to participate}

The research was undertaken in compliance with the ethical principles of the Helsinki Declaration and was approved by an institutional expert committee. Participants signed informed consent prior to the beginning of the test.

\section{Consent for publication}

All the authors approved the publication of the article.

Competing interests

The authors declare that they have no competing interests.

\section{Author details}

${ }^{1}$ Department of Information Engineering, Università Politecnica delle Marche, via Brecce Bianche, 60131 Ancona, Italy.

2 Laboratory of Bioengineering and Neuromechanics of Movement, University of Rome "Foro Italico", P.zza Lauro de Bosis 6, 00135 Rome, Italy.

Received: 8 January 2020 Accepted: 20 July 2020

Published online: 28 July 2020

\section{References}

1. Pacini Panebianco G, Bisi MC, Stagni R, Fantozzi S. Analysis of the performance of 17 algorithms from a systematic review: influence of sensor position, analysed variable and computational approach in gait timing estimation from IMU measurements. Gait Posture. 2018;66:76-82.

2. Taborri J, Palermo E, Rossi S, Cappa P. Gait partitioning methods: a systematic review. Sensors (Basel). 2016;16(1):66. https://doi.org/10.3390/s16010066.

3. Wentink E, Schut E, Prinsen E, Rietman E, Veltink P. Detection of the onset of gait initiation using kinematic sensors and emg in transfemoral amputees. Gait Posture. 2014;39(1):391-6.

4. Ferris D, Sawicki G, Daley M. A physiologist's perspective on robotic exoskeletons for human locomotion. Int J Humanoid Rob. 2007:4(3):507.

5. De Pauw K, Cherelle P, Roelands B, Lefeber D, Meeusen R. The efficacy of the ankle mimicking prosthetic foot prototype 4.0 during walking: physiological determinants. Prosthet Orthot Int. 2018:42(5):504-10.

6. McDonald C, Sullivan J, Dennis T, O'Malley M. A myoelectric control interface for upper-limb robotic rehabilitation following spinal cord injury. IEEE Trans Neural Syst Rehabil Eng. 2020;28(4):978-87.

7. Miller A. Gait event detection using a multilayer neural network. Gait Posture. 2009;29(4):542-5.

8. Osis ST, Hettinga BA, Ferber R. Predicting ground contact events for a continuum of gait types: an application of targeted machine learning using principal component analysis. Gait Posture. 2016;46:86-90.

9. Kidziński Ł, Delp S, Schwartz M. Automatic real-time gait event detection in children using deep neural networks. PLoS ONE. 2019;14(1):e0211466. https://doi.org/10.1371/journal.pone.0211466.eCollection.

10. Meng M, She Q, Gao Y, Luo Z. EMG signals based gait phases recognition using hidden Markov models. In: Proceedings of the 2010 IEEE international conference on information and automation, Harbin, China, 20-23 June 2010; pp. 852-856.

11. Nazmi N, Abdul Rahman M, Yamamoto Sl, Ahmad S. Walking gait event detection based on electromyography signals using artificial neural network. Biomed Signal Process Control. 2019;47:334-43.

12. Morbidoni C, Cucchiarelli A, Fioretti S, Di Nardo F. A deep learning approach to EMG-based classification of gait phases during level ground walking. Electronics. 2019;8(8):894. https://doi.org/10.3390/electronics8080894.

13. Mengarelli A, Maranesi E, Burattini L, Fioretti S, Di Nardo F. Co-contraction activity of ankle muscles during walking: a gender comparison. Biomed Signal Process Control. 2017;33:1-9.

14. Gurney J, Kersting U, Rosenbaum D. Between-day reliability of repeated plantar pressure distribution measurements in a normal population. Gait Posture. 2008;27:706-9.

15. Bovi G, Rabuffetti M, Mazzoleni P, Ferrarin M. A multiple-task gait analysis approach: kinematic, kinetic and EMG reference data for healthy young and adult subjects. Gait Posture. 2011;33:6-13.

16. Kamruzzaman J, Begg RK. Support vector machines and other pattern recognition approaches to the diagnosis of cerebral palsy gait. IEEE Trans Biomed Eng. 2006:53(12 Pt 1):2479-90.

17. Tang Z, Zhang K, Sun S, Gao Z, Zhang L, Yang Z. An upper-limb power-assist exoskeleton using proportional myoelectric control. Sensors (Basel). 2014;14(4):6677-94. https://doi.org/10.3390/s140406677.

18. Morbidoni C, Principi L, Mascia G, Strazza A, Verdini F, Cucchiarelli A, Di Nardo F. Gait phase classification from surface EMG signals using neural networks. In: Henriques J, Neves N, de Carvalho P (eds). XV mediterranean conference on medical and biological engineering and computing-MEDICON 2019. MEDICON 2019. IFMBE Proceedings, Springer, Cham. 2020;76:75-82.

19. Ziegier J, Gattringer H, Mueller A. Classification of gait phases based on bilateral EMG data using support vector machines. In Proceedings of the IEEE RAS and EMBS international conference on biomedical robotics and biomechatronics, Enschede, The Netherlands, 26-29 August. 2018:978-983.

20. Joshi CD, Lahiri U, Thakor NV. Classification of gait phases from lower limb EMG: Application toexoskeleton orthosis. In Proceedings of the 2013 IEEE Point-of-Care Healthcare Technologies (PHT), Bangalore, India, 16-18 January 2013:228-231.

21. Khandelwal S, Wickstrasm N. Evaluation of the performance of accelerometer-based gait event detection algorithms in different real-world scenarios using the MAREA gait database. Gait Posture. 2017;51:84-90. 
22. Caramia C, De Marchis C, Schmid M. Optimizing the scale of a wavelet-based method for the detection of gait events from a waist-mounted accelerometer under different walking speeds. Sensors. 2019;19(8):1869.

23. Flood M, O'Callaghan B, Lowery M. Gait event detection from accelerometry using the teager-kaiser energy operator. IEEE Trans Biomed Eng. 2020;67(3):658-66.

24. Trojaniello D, Cereatti A, Della Croce U. Accuracy, sensitivity and robustness of five different methods for the estimation of gait temporal parameters using a single inertial sensor mounted on the lower trunk. Gait Posture. 2014;40(4):487-92.

25. Judge JO, Davis RB, Ounpuu S. Step length reductions in advanced age: the role of ankle and hip kinetics. J Gerontol A Biol Sci Med Sci. 1996;51(6):M303-12.

26. Sofuwa O, Nieuwboer A, Desloovere K, Willems AM, Chavret F, Jonkers I. Quantitative gait analysis in parkinson's disease: comparison with a healthy control Group. Arch Phys Med Rehabil. 2005;86(5):1007-13.

27. Di Nardo F, Mengarelli A, Maranesi E, Burattini L, Fioretti S. Assessment of the ankle muscle co-contraction during normal gait: a surface electromyography study. J Electromyogr Kinesiol. 2015;25(2):347-54.

28. Agostini V, Nascimbeni A, Gaffuri A, Knaflitz M. Multiple gait patterns within the same winters class in children with hemiplegic cerebral palsy. Clin Biomech. 2015;30:908-14.

29. Castagneri C, Agostini V, Rosati S, Balestra G, Knaflitz M. Asymmetry index in muscle activations. IEEE Trans Neural Syst Rehabil Eng. 2019;27(4):772-9.

30. Ma Y, Xie S, Zhang Y. A patient-specific EMG-driven neuromuscularmodel for the potential use of human-inspired gait rehabilitation robots. Comput Biol Med. 2016;70:88-98.

31. Azimi V, Nguyen TT, Sharifi M, Fakoorian SA, Simon D. Robust ground reaction force estimation and control of lowerlimb prostheses: theory and simulation. IEEE Trans Syst Man Cyber Syst. 2018;99:1-12.

32. Watanabe T, Endo S, Morita R. Development of a prototype of portable FES rehabilitation system for relearning of gait for hemiplegic subjects. Healthc Technol Lett. 2016;3:284-9.

33. Lerner ZF, Board WJ, Browning RC. Effects of obesity on lower extremity muscle function during walking at two speeds. Gait Posture. 2014;39(3):978-84.

34. Hermens HJ, Freriks B, Merletti R, Ha"gg G, Stegeman DF, Blok J, et al. European recommendations for surface electromyography, SENIAM, vol. 8. Enschede (NL): Roessingh Research and Development; 1999.

35. Winter DA. Yack HJ EMG profiles during normal human walking: stride-to-stride and inter-subject variability. Electroencephalogr Clin Neurophysiol. 1987;67(5):402-11.

36. Yang JF. Winter DA Electromyographic amplitude normalization methods: improving their sensitivity as diagnostic tools in gait analysis. Arch Phys Med Rehabil. 1984;65(9):517-21.

37. Agostini V, Balestra G, Knaflitz M. Segmentation and classification of gait cycles. IEEE Trans Neural Syst Rehabil Eng. 2014;22(5):946-52.

38. Perry J. Gait analysis—normal and pathological function. New York: Slack Inc; 1992.

\section{Publisher's Note}

Springer Nature remains neutral with regard to jurisdictional claims in published maps and institutional affiliations.

- fast, convenient online submission

- thorough peer review by experienced researchers in your field

- rapid publication on acceptance

- support for research data, including large and complex data types

- gold Open Access which fosters wider collaboration and increased citations

- maximum visibility for your research: over 100M website views per year

At BMC, research is always in progress.

Learn more biomedcentral.com/submissions 